\title{
FUNÇÕES DE AFILAMENTO PARA PLANTIOS DESBASTADOS DE Pinus taeda
}

\author{
Mauro Yoshitani Junior ${ }^{1}$, Nelson Yoshihiro Nakajima ${ }^{2}$, Julio Eduardo Arce ${ }^{2}$, Sebastião do Amaral Machado², \\ João Paulo Druszcz ${ }^{3}$, Roberto Tuyoshi Hosokawa ${ }^{2}$, Anabel Aparecida de Mello ${ }^{4}$ \\ ${ }^{1}$ Eng. Florestal, M. Sc., Curitiba, PR, Brasil - mauroyoshitani@ yahoo.com.br \\ ${ }^{2}$ Eng. Florestal, Dr., Depto. de Ciências Florestais, UFPR, Curitiba, PR, Brasil - nelson.nakajima@ufpr.br; jarce@ufpr.br; \\ samachado@ufpr.br; roberto.hosokawa@pq.cnpq.br \\ ${ }^{3}$ Eng. Florestal, Doutorando em Ciências Florestais da UFPR, Curitiba, PR, Brasil - jpaulorz@yahoo.com.br \\ ${ }^{4}$ Eng $^{\mathrm{a}}$. Florestal, Dr ${ }^{\mathrm{a}}$., Depto. de Ciências Florestais, UFS, São Cristóvão, SE, Brasil - anabel_mello@yahoo.com.br
}

Recebido para publicação: 16/09/2009 - Aceito para publicação: 08/09/2011

\begin{abstract}
Resumo
O presente trabalho teve como objetivo avaliar a precisão de três funções de afilamento para estimar diâmetros e volumes ao longo do fuste de Pinus taeda L. A base de dados foi composta por 320 árvores cubadas pelo método de Smalian, sendo os diâmetros medidos nas alturas de 0,1 m, 0,5 m, $1,3 \mathrm{~m}, 2,0 \mathrm{~m}$ e, posteriormente, a cada $1,0 \mathrm{~m}$, até atingir a altura total. Essas árvores têm idades variando de 11 a 26 anos, podadas e com ocorrência de 2 a 6 desbastes até o corte final. Os modelos testados foram os de Schöepfer, Hradetzky e de Kozak et al. As análises estatísticas dos modelos testados foram realizadas através da análise gráfica dos resíduos, coeficiente de determinação ajustado $\left(\mathrm{R}^{2}\right)$, erro-padrão da estimativa $(\mathrm{Syx} \%)$ e análise gráfica dos perfis médios. Conclui-se que os modelos de Hradetzky e o de Shöepfer obtiveram resultados semelhantes e satisfatórios, com alto valor de coeficiente de determinação e baixo erro-padrão da estimativa, tanto para estimar diâmetros quanto volume, por serem modelos cujos parâmetros conseguem descrever melhor a extremidade superior e inferior da árvore.

Palavras-chave: Perfil do tronco; estimativa de volume; sortimento; plantios de Pinus.
\end{abstract}

\begin{abstract}
Taper function for thinning planting of Pinus taeda L.. This research aimed to evaluate accuracy of three taper functions in order to estimate diameters and volumes along the stem of Pinus taeda. The database consisted of 320 trees on which diameters were measured at heights of $0.1 \mathrm{~m}, 0.5 \mathrm{~m}, 1.3 \mathrm{~m}$, $2.0 \mathrm{~m}$, then at every $1.0 \mathrm{~m}$, until the total height. The age of these trees ranged from 11 to 26 years old and they were submitted to one pruning and thinned two to six times until the final cut. The tested models were those from Schöepfer, Hradetzky and Kozak et al.. The statistical analysis performed were graphical analysis of residuals, adjusted coefficient of determination $\left(\mathrm{R}^{2}\right)$, standard error of estimate (Syx\%), and graphical analysis of the mean profiles. As result, models from Hradetzky and Shöepfer obtained similar and satisfactory results, with high coefficient value of determination and low standard error of estimate, as to diameter as well to volume estimates; it is because these are models whose parameters can better describe both extremities of the tree.
\end{abstract}

Keywords: Stem profile; volume estimation; assortment; Pinus plantation.

\section{INTRODUÇÃO}

Nas últimas duas décadas, com o avanço tecnológico, houve também uma grande evolução da ciência florestal brasileira, o que propiciou a revisão de uma série de conceitos no que concerne ao uso do recurso florestal. No Brasil, a tecnologia de plantios florestais relativa aos gêneros Pinus e Eucalyptus é bastante desenvolvida, com a destinação da produção bem definida, enquanto que nas florestas nativas há ainda muito que se conhecer sobre as espécies que a compõem, como crescem em comunidade ou em plantios homogêneos, qual o potencial para aproveitamento econômico ou ecológico etc.

O manejo florestal é uma prática em que o objetivo maior é aumentar a qualidade do produto final, sua dimensão e se possível a sua quantidade, observando em todas as fases a viabilidade 
socioeconômica e ambiental do processo produtivo. Um segundo enfoque considera o manejo florestal como um processo de tomada de decisão. Nesse contexto, o profissional florestal necessita ter uma visão global de planejamento florestal, utilizando para tal modelos matemáticos que possibilitem a previsão da produção, assim como o gerenciamento de toda essa gama de informações, por meio de planos de manejo em que a otimização seja a tônica do processo. Naturalmente que, seja em cultivos florestais, seja em florestas, o manejador florestal deve balizar suas decisões em informações biológicas, econômicas, sociais e ambientais, de modo que a sustentabilidade dessa prática perpetue a atividade florestal no local onde o empreendimento estiver sendo executado.

A necessidade de boas estimativas de volumes comerciais nos cultivos florestais tem despertado o interesse pelo uso das funções de afilamento. Essas funções permitem a estimativa do diâmetro em uma altura qualquer do fuste, da altura comercial para um dado diâmetro, ou, ainda, do volume até um diâmetro mínimo qualquer. Resultados mais elaborados de inventário têm sido obtidos, enriquecendo a tradicional informação de volume total e volume por unidade de área.

A precisão da avaliação quantitativa de estoques florestais é de fundamental importância no sentido de direcionar a matéria-prima para diferentes usos. A partir de informações provenientes de inventários florestais, é possível definir um plano de manejo visando à utilização de usos múltiplos da floresta, ou seja, obter os volumes comerciais e ainda volumes de partes específicas do fuste. Uma vez realizada a qualificação e quantificação dos produtos madeiráveis das árvores (volumes comerciais), evidencia-se a potencialidade dos cultivos florestais para uma combinação de usos de material lenhoso, de suma importância no planejamento florestal.

O emprego desses modelos é uma consequência natural da evolução e importância do setor florestal no Brasil, no qual o aprimoramento das técnicas de inventário, associado à necessidade de flexibilização da informação de estoques de madeira, despertou um maior interesse na depuração das informações de inventário.

Para expressar a forma do tronco de árvores, a equação de afilamento é o modo mais difundido. Segundo Ahrens e Holbert (1981), uma função de afilamento é uma descrição matemática do perfil longitudinal de um tronco, e o seu volume pode ser obtido por integração dessa função e, assim, em termos geométricos, o tronco é tratado como um sólido de revolução. Uma vez definido um modelo matemático para forma, pode-se determinar o volume de madeira entre quaisquer pontos ao longo do tronco. Do ponto de vista dendrométrico, a forma do fuste de árvores genericamente é sua configuração externa que, a rigor, não se identifica com a forma de um sólido geométrico específico, mas sim com vários, segundo a porção do tronco considerada. De maneira mais restrita, a forma do fuste pode ser associada ao termo afilamento, que representa a diminuição do diâmetro com o aumento da altura, tendência natural que ocorre na maioria das espécies e como tal pode ser expressa por uma função matemática que descreve essa tendência de variação. Afilamento do tronco ("taper") tem sido definido como o decréscimo em diâmetro da base para sua extremidade superior. As funções de afilamento permitem a estimativa do diâmetro numa altura qualquer do fuste, da altura comercial para um dado diâmetro superior, de um volume até um diâmetro mínimo qualquer ou ainda de qualquer segmento ao longo do fuste.

Conforme Ferreira (2004), as funções de afilamento também permitem obter, por integração, o volume total e os volumes de partes do tronco. Dentre as principais funções de afilamento, existem aquelas que merecem destaque, por intensidade de utilização e, ou, por comprovada acurácia, como os modelos sigmoidais, polinomiais e os polinomiais segmentados.

Nesse contexto, levando em consideração os dados de cubagem rigorosa, o objetivo desta pesquisa foi ajustar os modelos de polinômio de $5^{\circ}$ grau (SHÖEPFER, 1966), polinômio de potências fracionárias (HRADETZKY, 1976) e o modelo de Kozak et al. (1969) e indicar qual o modelo mais adequado e preciso para estimar o(s) diâmetro(s) e volume(s) ao longo do fuste, sob a hipótese de que a escolha do modelo mais preciso seja feita através da análise estatística do coeficiente de determinação $\left(\mathrm{R}^{2}\right)$, do erro-padrão da estimativa (Syx\%) e da análise gráfica de resíduos, os quais são analisados conjuntamente para o aval final.

\section{MATERIAL E MÉTODOS}

\section{Equações de afilamento}

Polinômio de quinto grau - Schöepfer (1966)

O polinômio de quinto grau foi ajustado por classe diamétrica e tem a forma: 


$$
\frac{d_{i}}{D A P}=\beta_{0}+\beta_{1}\left(\frac{h_{i}}{H}\right)+\beta_{2}\left(\frac{h_{i}}{H}\right)^{2}+\beta_{3}\left(\frac{h_{i}}{H}\right)^{3}+\beta_{4}\left(\frac{h_{i}}{H}\right)^{4}+\beta_{5}\left(\frac{h_{i}}{H}\right)^{5}+e_{i}
$$

em que: $d_{i}=$ diâmetro estimado $(\mathrm{cm})$;

$h_{i}=$ altura qualquer $(\mathrm{m})$;

$\beta_{i}=$ parâmetros a serem estimados;

$D A P=$ diâmetro à altura do peito $(\mathrm{cm})$;

$H=$ altura total $(\mathrm{m})$;

$e_{i}=$ erro de estimativa.

Isolando $\mathrm{d}_{\mathrm{i}}$, obtém-se a função de afilamento através da qual se pode estimar o diâmetro correspondente a qualquer altura na árvore, desde que fornecido o DAP e a altura total.

$$
d_{i}=D A P\left[\beta_{0}+\beta_{1}\left(\frac{h_{i}}{H}\right)+\beta_{2}\left(\frac{h_{i}}{H}\right)^{2}+\beta_{3}\left(\frac{h_{i}}{H}\right)^{3}+\beta_{4}\left(\frac{h_{i}}{H}\right)^{4}+\beta_{5}\left(\frac{h_{i}}{H}\right)^{5}\right]
$$

O volume de cada árvore foi obtido pela integral do polinômio de quinto grau - Schöepfer (1966):

$$
v=k \cdot D A P^{2}\left[\begin{array}{l}
b_{0}{ }^{2} x_{1}+\left(b_{0} b_{1} x_{1}{ }^{2}\right)+\left(\frac{2}{3} b_{0} b_{2}+\frac{1}{3} b_{1}{ }^{2}\right) x 13+\left(\frac{1}{2} b_{0} b_{3}+\frac{1}{2} b_{1} b_{2}\right) x_{1}{ }^{4} \\
+\left(\frac{2}{5} b_{0} b_{4}+\frac{2}{5} b_{1} b_{3}+\frac{1}{5} b_{2}{ }^{2}\right) x_{1}{ }^{5}+\left(\frac{1}{3} b_{0} b_{3}+\frac{1}{3} b_{1} b_{4}+\frac{1}{3} b_{1} b_{2}\right) x_{1}{ }^{6}+ \\
\left.\frac{2}{7} b_{1} b_{5}+\frac{2}{7} b_{2} b_{4}+\frac{1}{7} b_{3}{ }^{2}\right) x_{1}{ }^{7}+\left(\frac{1}{4} b_{2} b_{5}+\frac{1}{4} b_{3} b_{4}\right) x_{1}{ }^{8}+\left(\frac{2}{9} b_{3} b_{5}+\frac{1}{9} b_{4}{ }^{2}\right) x_{1}{ }^{9}+ \\
\frac{1}{5} b_{4} b_{5} x_{1}{ }^{10}+\frac{1}{11} b_{5}{ }^{2} x_{1}{ }^{11}
\end{array}\right]_{h 1}^{h 2}
$$

em que: $k=\pi / 40000$;

$x_{1}=(\mathrm{hi} / \mathrm{H})$

$h_{i}, H, D A P, \beta_{i}$ e $e_{i}=$ definidos anteriormente.

Polinômio de potência fracionária - Hradetzky (1976)

Esse modelo se diferencia do polinômio do quinto grau por apresentar um mix de potências inteiras e fracionárias, como tentativa de melhor representar o perfil da árvore. Hradetzky (1976) sugeriu a utilização de potências inteiras da ordem de dezenas para representar a base da árvore, em conjunto com potências fracionárias para representar a porção superior do fuste. Esses modelos foram construídos por classe diamétrica, utilizando-se o procedimento "stepwise".

Os expoentes utilizados na construção dos modelos por classe de diâmetro por meio do procedimento de "stepwise" foram: 0,$00001 ; 0,00005 ; 0,0001 ; 0,0002 ; 0,0004 ; 0,0006 ; 0,0007 ; 0,0008$; 0,$0009 ; 0,005 ; 0,09 ; 0,08 ; 0,07 ; 0,06 ; 0,05 ; 0,04 ; 0,03 ; 0,02 ; 0,01 ; 0,9 ; 0,8 ; 0,7 ; 0,6 ; 0,5 ; 0,4 ; 0,3 ; 0,2 ; 0,1$; $1 ; 2 ; 3 ; 4 ; 5 ; 10 ; 15 ; 20 ; 25 ; 30 ; 35 ; 40 ; 45 ; 50 ; 55 ; 60 ; 65 ; 70 ; 75 ; 85 ; 90$ e 95 .

De forma geral, os polinômios a serem construídos são:

$$
\frac{d_{i}}{D A P}=\beta_{0}+\beta_{1}\left(\frac{h_{i}}{H}\right)^{p 1}+\beta_{2}\left(\frac{h_{i}}{H}\right)^{p 2}+\ldots+\beta_{n}\left(\frac{h_{i}}{H}\right)^{p n}+e_{i}
$$

em que: $d_{i}, D, h_{i}, H, \beta_{i}$, e $e_{i}=$ definidos anteriormente;

$p_{i}=$ expoentes variando entre 0,00001 e 95 . 
Isolando $\mathrm{d}_{\mathrm{i}}$, obtém-se a função de afilamento:

$$
d_{i}=D A P\left[\beta_{0}+\beta_{1}\left(\frac{h_{i}}{H}\right)^{p 1}+\beta_{2}\left(\frac{h_{i}}{H}\right)^{p 2}+\ldots+\beta_{n}\left(\frac{h_{i}}{H}\right)^{p n}\right]
$$

O volume de cada árvore foi obtido pela integral do polinômio de potência fracionária Hradetzky (1976). A integral do modelo assume a forma:

$$
v=k \cdot D A P^{2}\left[\begin{array}{l}
b_{0}{ }^{2} x_{1}+2 b_{0} b_{1}\left(\frac{x_{1}^{1,005}}{1,005}\right)+\left(2 b_{0} b_{2}\left(\frac{x_{1}^{1,08}}{1,08}\right)+b_{1}^{2}\left(\frac{x_{1}^{1,01}}{1,01}\right)+2 b_{0} b_{3}\left(\frac{x_{1}^{3}}{3}\right)+\right. \\
+\left(2 b_{1} b_{2}\left(\frac{x_{1}^{1,805}}{1,805}\right)+2 b_{0} b_{4} \frac{x_{1}^{26}}{26}+2 b_{1} b_{3}\left(\frac{x_{1}^{3,005}}{3,005}\right)+b_{2}{ }^{2} \frac{x_{1}^{2,6}}{2,6}+2 b_{1} b_{4}\left(\frac{x_{1}^{26,005}}{26,005}\right)+\right. \\
2 b_{2} b_{3}\left(\frac{x_{1}^{13,8}}{3,8}\right)+2 b_{2} b_{4}\left(\frac{x_{1}^{26,8}}{26,8}\right)+b_{3}{ }^{2}\left(\frac{x_{1}{ }^{5}}{5}\right)+2 b_{3} b_{4}\left(\frac{x_{1}{ }^{28}}{28}\right)+b_{4}{ }^{2}\left(\frac{x_{1}{ }^{51}}{51}\right)
\end{array}\right]_{h 1}^{h 2}
$$

Polinômio de segundo grau ou modelo de Kozak et al. (1969)

$$
\left(\frac{d_{i}}{D A P}\right)^{2}=b_{0}+b_{1}\left(\frac{h_{i}}{H}\right)+b_{2}\left(\frac{h_{i}}{H}\right)^{2}
$$

em que: $d_{i}=$ diâmetro estimado;

$h i=$ altura qualquer;

$\beta_{i}=$ parâmetros a serem estimados;

$D A P=$ diâmetro à altura do peito;

$H=$ altura total;

$e_{i}=$ erro de estimativa.

O volume de cada árvore foi obtido pela integral do modelo de Kozak et al. (1969).

$$
v=k \cdot D A P^{2}\left(b_{0}\left(h_{2}-h_{1}\right)+\frac{b_{1}\left(h_{2}{ }^{2}-h_{1}{ }^{2}\right)}{2 h}+\frac{b_{2}\left(h_{2}{ }^{3}-h_{1}{ }^{3}\right)}{3 h^{2}}\right)
$$

\section{RESULTADOS E DISCUSSÃO}

\section{Ajustes dos modelos}

Após o ajuste dos três modelos de afilamento para diâmetro estimado, foram realizadas as análises estatísticas de coeficiente de determinação ajustado $\left(\mathrm{R}^{2}\right)$ e erro-padrão da estimativa (Syx\%) (Tabela 1).

Os modelos testados apresentaram coeficiente de determinação ajustado $\left(R^{2}\right)$ entre $88,85 \%$ (KOZAK et al., 1969) e 98,23\% (Hradetzky) e erro-padrão da estimativa (Syx\%) entre 7,92\% (Hradetzky) e 11,59\% (KOZAK et al., 1969). Para o conjunto de dados, somente os modelos de Shöepfer e Hradetzky apresentaram bom desempenho, enquanto o modelo de Kozak et al. (1969) obteve menor coeficiente de determinação ajustado e maior erro-padrão da estimativa, não sendo recomendado o seu uso para estimativa de diâmetro ao longo do fuste de Pinus taeda.

Todos os modelos apresentaram alguma tendenciosidade ao longo da linha média, e os maiores erros ocorreram na porção inferior do fuste (Figura 1).

O modelo de potências fracionárias de Hradetzky foi o que melhor estimou o diâmetro ao longo do fuste, apresentando maior coeficiente de determinação $(98,23 \%)$, menor erro-padrão da estimativa $(7,92 \%)$ e menor tendenciosidade na análise gráfica dos resíduos. O modelo de Kozak et al. (1969) foi o que apresentou pior desempenho, com menor coeficiente de determinação $(88,85 \%)$ e maior erro-padrão da estimativa $(11,59 \%)$, subestimando muito os diâmetros ao longo do fuste. 
Os resultados das análises estatísticas indicam que os modelos de Shöepfer e Hradetzky apresentaram os melhores ajustes, mas com alguma tendenciosidade nos menores diâmetros. Já o ajuste do modelo de Kozak et al. (1969) foi relativamente inferior.

Tabela 1. Coeficiente de regressão, coeficiente de determinação e erro-padrão da estimativa de cada modelo de afilamento para estimativa de diâmetros ao longo do fuste.

Table 1. Regression coefficient, determination coefficient and standard error of estimate for each tapper function to estimate diameter along the stem.

\begin{tabular}{|c|c|c|c|c|c|c|c|c|}
\hline \multicolumn{9}{|c|}{ Shöepfer } \\
\hline$\beta_{0}$ & $\beta_{1}$ & $\beta_{2}$ & $\beta_{3}$ & $\beta_{4}$ & $\beta_{5}$ & $\mathrm{R}^{2}$ aj. (\%) & $\begin{array}{l}\text { Syx } \\
(\%)\end{array}$ & Syx $(\mathrm{cm})$ \\
\hline 1,19054 & $-3,98912$ & 17,8176 & $-38,203$ & 35,6996 & $-12,51$ & 97,29 & 8,10 & 1,81 \\
\hline \multicolumn{9}{|c|}{ Hradetzky } \\
\hline$\beta_{0}$ & $\begin{array}{l}\beta_{1} \\
(1)\end{array}$ & $\begin{array}{l}\beta_{2} \\
(4)\end{array}$ & $\begin{array}{l}\beta_{3} \\
(5)\end{array}$ & $\begin{array}{c}\beta_{4} \\
(95)\end{array}$ & $\beta_{5}(0,009)$ & $\begin{array}{l}\mathrm{R}^{2} \text { aj. } \\
(\%)\end{array}$ & $\begin{array}{l}\text { Syx } \\
(\%)\end{array}$ & Syx $(\mathrm{cm})$ \\
\hline 1,24288 & 0,125188 & $-2,75867$ & 2,04837 & $-0,1071$ & $-11,73$ & 98,23 & 7,92 & 1,77 \\
\hline \multicolumn{9}{|c|}{ Kozak et al. } \\
\hline$\beta_{0}$ & $\beta_{1}$ & $\beta_{2}$ & & & & $\begin{array}{c}\mathrm{R}^{2} \text { aj. } \\
(\%)\end{array}$ & $\begin{array}{l}\text { Syx } \\
(\%)\end{array}$ & Syx $(\mathrm{cm})$ \\
\hline 1,1995 & $-1,78064$ & 0,600837 & & & & 88,85 & 11,59 & 2,59 \\
\hline
\end{tabular}
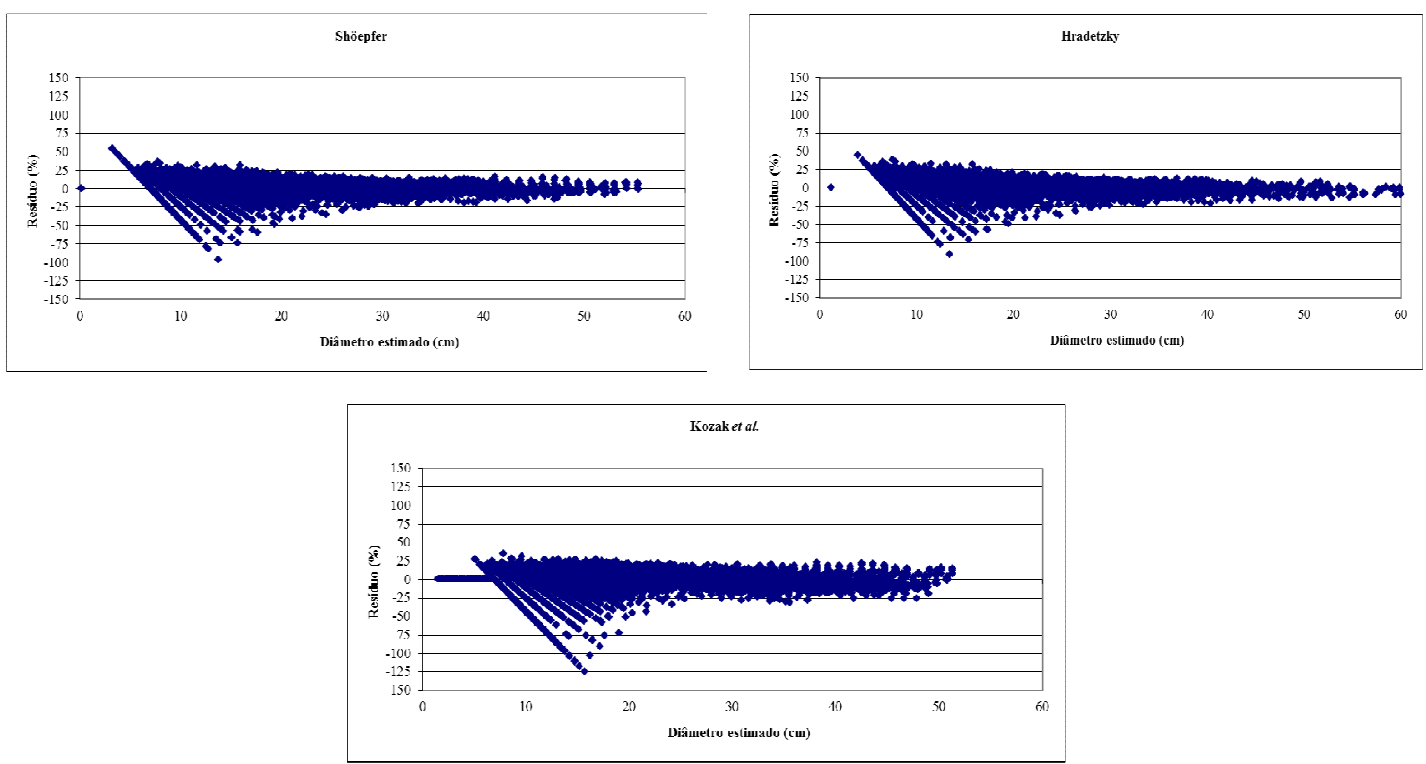

Figura 1. Distribuição dos resíduos (\%) em função do diâmetro estimado $(\mathrm{cm})$ para os três modelos de afilamento.

Figure 1. Distribution of residuals $(\%)$ according to estimated diameter $(\mathrm{cm})$ for three tapper function.

\section{Perfil médio dos fustes}

A figura 2 apresenta o perfil médio dos fustes estimados pelos três modelos de afilamento testados para Pinus taeda com casca.

Pela análise gráfica do perfil do fuste, pode-se confirmar a superioridade do modelo polinomial de Hradetzky, pois ele não apresentou tendenciosidade na descrição do perfil dos fustes estimados. Conforme a figura 2, o polinômio de quinto grau apresentou pequena subestimativa nos menores diâmetros. Na posição de 0,5 a 0,8 , representou bem o perfil do fuste, e de 0,8 em diante novamente teve pequena subestimativa. 


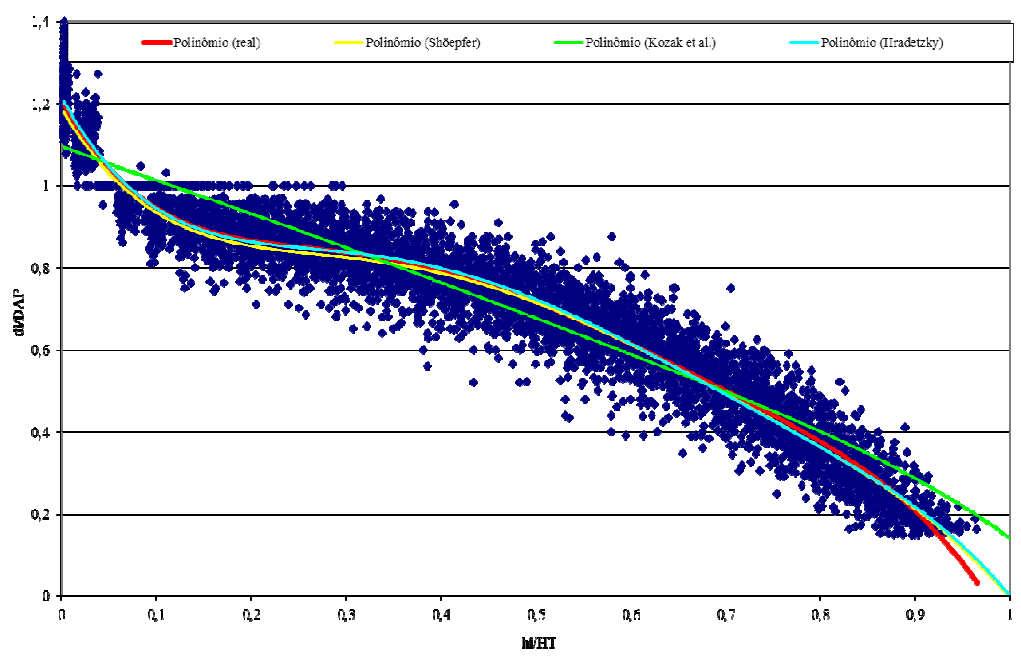

Figura 2. Perfil médio dos fustes estimados pelos modelos de Shöepfer (1966), Kozak et al. (1969) e Hradetzky (1979) para Pinus taeda.

Figure 2. Average profile of the stem estimated by the models of Shöepfer (1966), Kozak et al. (1969) and Hradetzky (1979) for Pinus taeda.

Já o polinômio de Kozak et al. (1969) apresentou maior tendenciosidade, superestimando ou subestimando o diâmetro, confirmando o resultado das análises estatísticas e análise gráfica dos resíduos, mostrando-se inadequado para estimar diâmetros em diferentes alturas em povoamento de Pinus taeda. Fisher (1997), que estudou a eficiência dos modelos polinomiais e das razões de volume na estimativa volumétrica dos sortimentos e do perfil dos fustes de Pinus taeda, recomenda a utilização do modelo polinomial de potências fracionárias com ajuste por sítio e por classe diamétrica para descrever o perfil do tronco.

\section{Avaliação dos modelos polinomiais para estimativa dos volumes}

Na tabela 2 são mostrados os erros-padrão da estimativa em porcentagem (Syx\%) para a estimativa do volume. Esses erros foram calculados a partir da amostra das árvores que foram cubadas pelo método de Smalian. Os resultados mostraram valores consideráveis para o modelo de Shöepfer $(11,80 \%)$, Hradetzky $(12,85 \%)$ e de Kozak et al. $(14,88 \%)$, sendo que este último não apresentou uma boa precisão em relação aos outros dois modelos testados.

Tabela 2. Coeficiente de determinação e erro-padrão da estimativa de cada modelo de afilamento para volume estimado.

Table 2. Coefficient of determination and mean standard error for each tapper function to estimate volume.

\begin{tabular}{lcc}
\hline \multicolumn{3}{c}{ Shöepfer } \\
\hline $\mathrm{R}^{2}$ aj.(\%) & Syx (\%) & Syx $\left(\mathrm{m}^{3}\right)$ \\
97,29 & 11,80 & 0,11534 \\
\hline \multicolumn{3}{c}{ Hradetzky } \\
\hline $\mathrm{R}^{2}$ aj.(\%) & Syx (\%) & Syx $\left(\mathrm{m}^{3}\right)$ \\
98,23 & 12,85 & 0,12559 \\
\hline \multicolumn{3}{c}{ Kozak et al. } \\
\hline $\mathrm{R}^{2}$ aj.(\%) & Syx $(\%)$ & Syx $\left(\mathrm{m}^{3}\right)$ \\
88,85 & 14,25 & 0,13929 \\
\hline
\end{tabular}

Na figura 3, é apresentada a análise gráfica dos resíduos (\%) em função do volume estimado $\left(\mathrm{m}^{3}\right)$. Pode-se observar alguma tendenciosidade na estimativa do volume $\left(\mathrm{m}^{3}\right)$. Nota-se que todos os modelos apresentaram tendenciosidade quanto à estimativa do volume. Novamente, o modelo polinomial de potência fracionária de Hradetzky apresentou o melhor desempenho. 

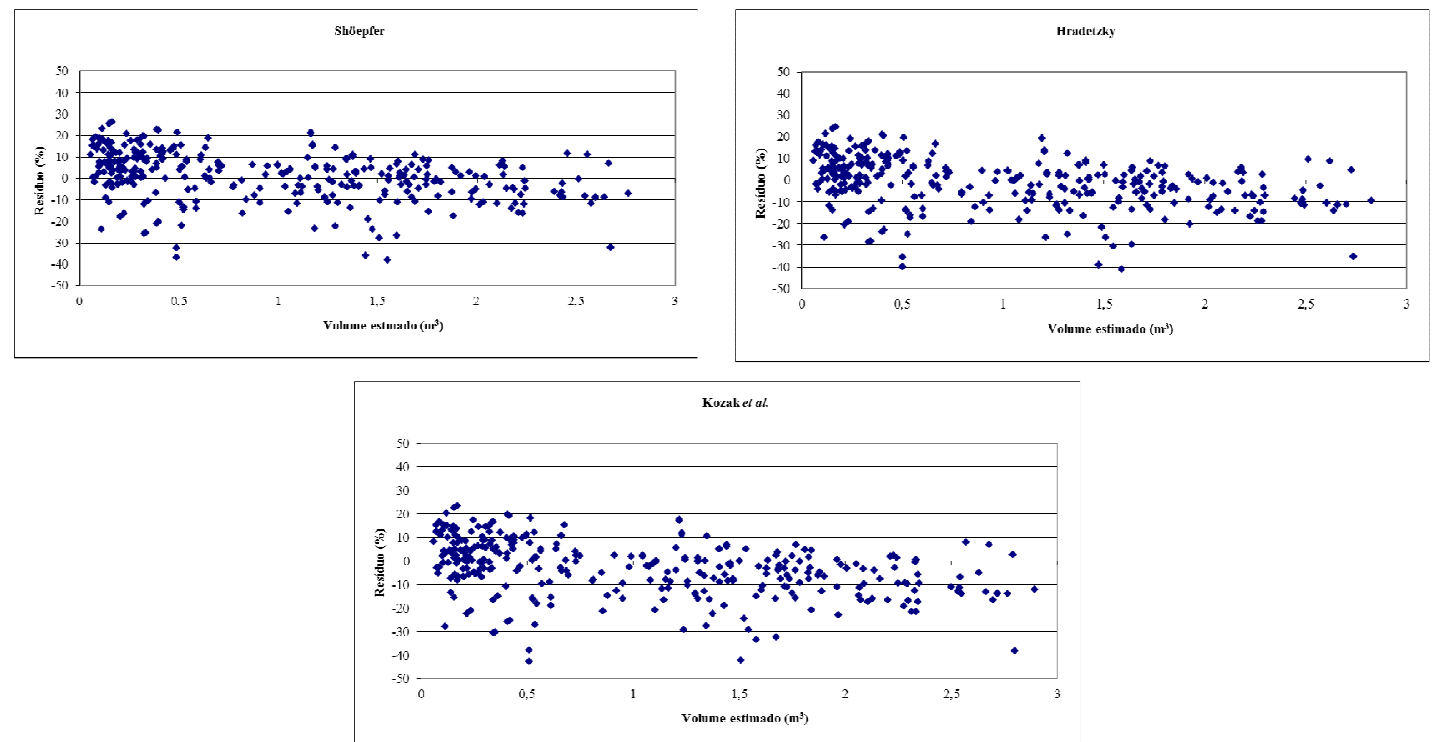

Figura 3. Distribuição gráfica dos resíduos (\%) para os três modelos testados.

Figure 3. Graphic distribution of residuals (\%) for the three tested models.

$\mathrm{Na}$ figura 4, também se pode observar ligeira vantagem dos modelos polinomiais de quinto grau e de potências fracionárias, pois esses modelos são flexíveis o suficiente para descrever a variação da forma da árvore. Novamente o modelo de Kozak et al. (1969) apresentou as piores estimativas, com tendência de subestimativa.
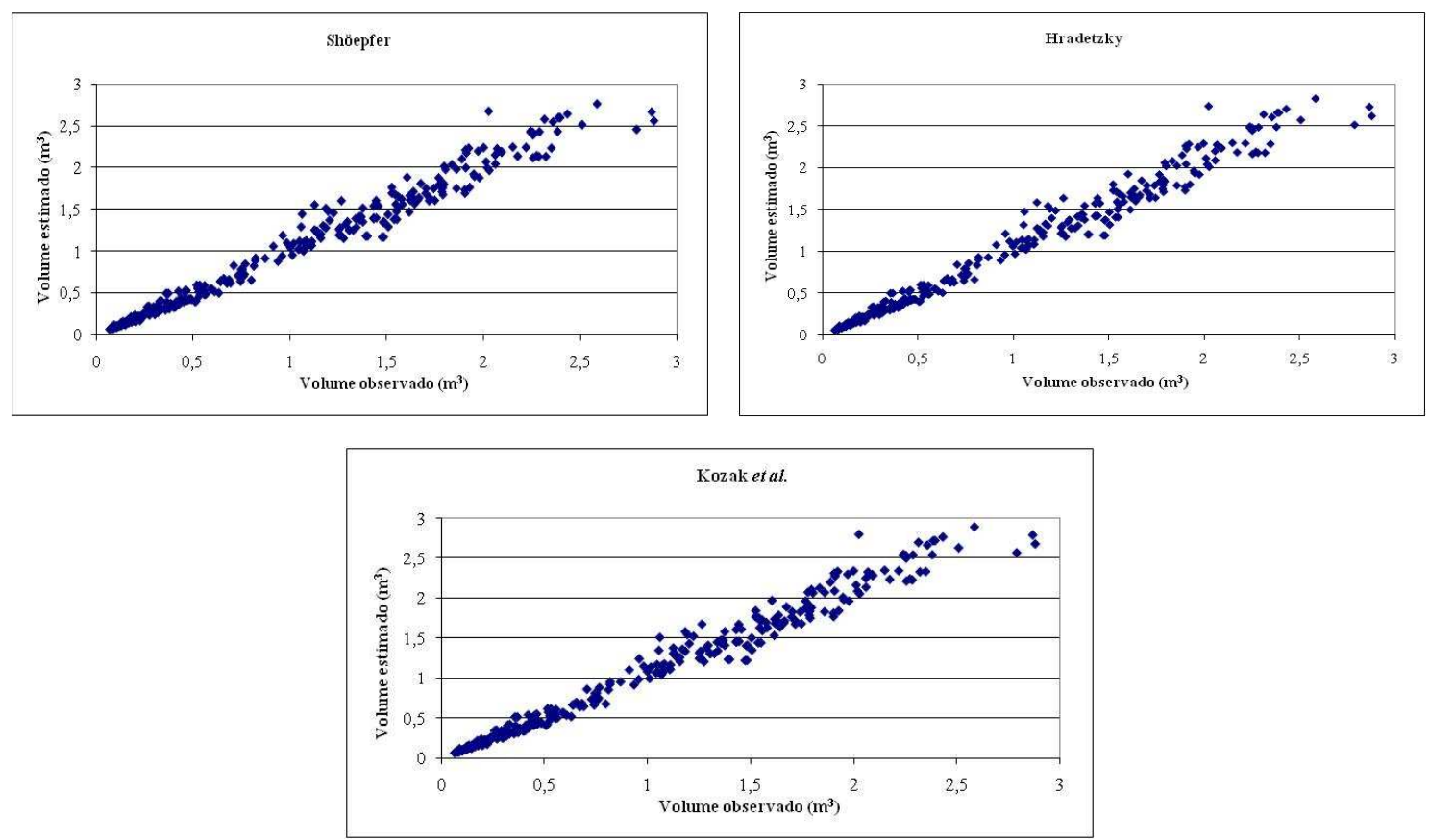

Figura 4. Volume estimado $\left(\mathrm{m}^{3}\right)$ pelas equações de afilamento de Shöepfer, Hradetzky e Kozak et al. sobre os correspondentes valores observados $\left(\mathrm{m}^{3}\right)$.

Figure 4. Estimate volume $\left(\mathrm{m}^{3}\right)$ for the tapper models of Shöepfer, Hradetzky and Kozak et al. on the corresponding observed values $\left(\mathrm{m}^{3}\right)$. 
Assis (2002) testou o polinômio de quinto grau, o polinômio de Hradetzky (1976) e o polinômio de Golding e Hall em árvores de Pinus taeda, em diferentes ambientes de produção, e constatou que o modelo de Hradetzky (1976) foi o melhor na estimativa dos diâmetros em todos eles. Para a estimativa dos volumes, o melhor modelo na maioria dos ambientes foi o modelo de quinto grau. Machado et al. (2004), também trabalhando com árvores de Pinus oocarpa em várias classes de idades e sujeitas a diferentes números de desbastes, observaram que, para a estimativa de diâmetros com e sem casca, ao longo do fuste, o modelo de Hradetzky (1976) apresentou, em média, o melhor desempenho, seguido do modelo de Shöepfer.

\section{CONCLUSÕES}

- As equações polinominais de Shöepfer e Hradetzky propiciam estimativas precisas do diâmetro e volume, com coeficiente de determinação, erro-padrão da estimativa e análise gráfica de resíduos muito próximos.

- O modelo de Kozak et al. apresenta as piores estatísticas e teve o pior desempenho para estimar o diâmetro e volume para o conjunto de dados, não sendo então priorizado o seu uso para as condições do povoamento de Pinus taeda pesquisadas. Isso ocorre porque os parâmetros desse modelo não conseguem descrever as extremidades superior e inferior da árvore, ou seja, não é um modelo flexível para essas partes da árvore.

- Os modelos de Shöepfer e Hradetzky apresentaram melhor precisão, por serem mais flexíveis para estimar a variação da forma do fuste de uma árvore, porque seus parâmetros conseguem descrever melhor as extremidades superior e inferior da árvore.

\section{REFERÊNCIAS}

AHRENS, S.; HOLBERT, D. Uma função para forma de tronco e volume de Pinus taeda L. In: Boletim de Pesquisa Florestal, unidade regional de pesquisa florestal Centro-Sul, EMBRAPA, v. 3, p. 37 - 68, 1981.

ASSIS, A. L.; SCOLFORO, J. R. S.; MELLO, J. M.; OLIVEIRA, A. D. Avaliação de modelos polinomiais não segmentados na estimativa de diâmetros e volumes comerciais de Pinus taeda. Ciência Florestal, Santa Maria, v. 12, n. 1, p. 89 - 108, 2002.

FERREIRA, M. Z. Estudo de funções de afilamento para representar o perfil e o volume do fuste de Pinus taeda L. 196 f. Dissertação (Mestrado em Engenharia Florestal) - Universidade Federal de Lavras, Lavras, 2004.

FISHER, F. Eficiência dos modelos polinomiais e das razões de volume na estimativa volumétrica dos sortimentos e do perfil do fuste de Pinus taeda. 167 f. Dissertação (Mestrado em Engenharia Florestal) - Universidade Federal de Lavras, Lavras, 1997.

HRADETZKY, J. Analysis und interpretation statistisher abränger keiten (Biometrische Beiträge zu aktuellen forschungs projekten). Baden - Württemberg Mitteilungen der FVA, Helf 76, 1976. 146 p. (Abt. Biometric und Informatik, 21).

KOZAK, A.; MUNRO, D. P.; SMITH, J. H. G. Taper functions and their application in forest inventory. Forest Chronicle, Toronto, v. 45, n. 4, p. 278 - 283, 1969.

MACHADO, S. A.; URBANO, E.; CONCEIÇÃO, M. B.; FIGUEIREDO, D. J. Comparação de modelos de afilamento do tronco para diferentes idades e regimes de desbaste em plantações de Pinus oocarpa Schiede. In: Boletim de Pesquisa Florestal, Colombo, n. 48, p. 41 - 64, 2004.

SCHÖEPFER, W. Automatisierrung des massen, Sorten und Wertberechnung stenender Waldbestande Schriftenreihe Bad. [S.1]: Wurtt-Forstl., 1966. n.p. 